\title{
Ultraviolet poling of pure fused silica by high-intensity femtosecond radiation
}

\author{
Costantino Corbari and Peter G. Kazansky \\ Optoelectronics Research Centre, University of Southampton, Southampton, United Kingdom \\ Stephen A. Slattery and David N. Nikogosyan ${ }^{\text {a) }}$ \\ Physics Department, National University of Ireland, University College Cork, Cork, Ireland
}

(Received 27 October 2004; accepted 30 December 2004; published online 8 February 2005)

\begin{abstract}
We demonstrate UV poling of a pure fused silica sample by applying to it an electric field of 200
$\mathrm{kV} / \mathrm{cm}$ and irradiating it with high-intensity $\left(\sim 40 \mathrm{GW} / \mathrm{cm}^{2}\right)$ femtosecond $(220 \mathrm{fs})$ laser pulses at 264 nm. () 2005 American Institute of Physics. [DOI: 10.1063/1.1868075]
\end{abstract}

The possibility of inducing a second-order nonlinearity in glass by poling has some very intriguing possibilities for fiber optics in particular and photonics in general, as it has opened the way for creating different built-in devices in a standard telecom fiber; amongst them modulators, switches, and harmonic generators. The method of UV poling, first realized by Fujiwara et al., ${ }^{1}$ consists of the irradiation of highly Ge-doped $(15.7 \mathrm{~mol} \%)$ fused silica glass by nanosecond $193 \mathrm{~nm}$ laser pulses in the presence of an applied electric field of 100-300 kV/cm. Fujiwara et al. reported the induction of a nonlinear second-order coefficient as high as 3.4 $\mathrm{pm} / \mathrm{V}$. Despite his following publications on this subject, ${ }^{2-4}$ there is growing skepticism about these results in scientific literature, ${ }^{5,6}$ as they are not reproducible (maybe due to a special nature of the sample used by him). In this investigation, we tried to repeat Fujiwara's experiments using a highintensity UV femtosecond laser source and undoped fused silica samples, excited by a two-photon mechanism. ${ }^{7,8}$ After irradiation, we observed a strong second-harmonic (SH) signal in the areas of the sample that had been subjected both to the electric field and to the UV light.

The experimental setup is shown in Fig. 1. The fused silica samples used for poling were made from Herasil 1 glass (Heraeus Quarzglas $\mathrm{GmbH}$ ) and had dimensions 37 $\times 11 \times 0.5 \mathrm{~mm}^{3}$. All the sides of the samples were polished and aluminum electrodes, $11 \times 5 \mathrm{~mm}^{2}$, were deposited on both plane sides of the samples in such a way that the edges of each electrode were at a distance of about $3 \mathrm{~mm}$ from the end of the plate and side edges (see Fig. 1).

For sample irradiation, we used the UV pulses from a femtosecond Nd:glass laser (Twinkle, Light Conversion Ltd., Vilnius, Lithuania) ${ }^{9}$ with the following parameters: the pulse energy, $\varepsilon_{p}$, is around $200 \mu \mathrm{J}$; the wavelength, $\lambda$, is $264 \mathrm{~nm}$; the pulse duration, $\tau$, is $220 \mathrm{fs}$ [full width at half maximum (FWHM)]; the beam diameter, $w$, is $0.3 \mathrm{~cm}(\mathrm{FWHM})$; and the repetition rate, $\Delta f$, is $27 \mathrm{~Hz}$. The $\mathrm{UV}$ radiation was focused into the narrow side of the sample by a cylindrical lens with focal length $21.8 \mathrm{~cm}$. The sample was placed at a distance of $21 \mathrm{~cm}$ from the lens. The laser beam size at the entrance of the sample was about $3 \times 0.15 \mathrm{~mm}^{2}$ (at FWHM). A high voltage block FC30P4 (Glassman High Voltage, Inc.) was used to create an electric field of about $200 \mathrm{kV} / \mathrm{cm}$ through the sample.

\footnotetext{
a) Author to whom correspondence should be addressed; electronic mail: niko@physics.ucc.ie
}

The peak beam intensity and the incident fluence were calculated from the following: ${ }^{8}$

$$
\begin{aligned}
& I=\frac{\varepsilon_{p}}{\left(\frac{\pi}{\ln 2}\right)^{3 / 2} \frac{\tau w^{2}}{4}\left(\frac{F-S}{F}\right)}, \\
& E=\frac{\varepsilon_{p} N}{\frac{\pi w^{2}}{2 \ln 2}\left(\frac{F-S}{F}\right)},
\end{aligned}
$$

where $\varepsilon_{p}$ is the pulse energy incident on the sample; $F$ is the focal distance of the lens at $264 \mathrm{~nm}$; $S$ is the distance between the lens and the sample (more precisely, between the principal plane of the lens and the sample); and $N$ is the total number of pulses incident on the sample. With the high voltage applied across the sample, the sample was irradiated with the high-intensity UV laser light for approximately $3 \mathrm{~h}$ (total incident fluence of around $8 \mathrm{~kJ} / \mathrm{cm}^{2}$ ) with peak incident intensity of around $200 \mathrm{GW} / \mathrm{cm}^{2}$ (at the narrow edge of the sample). Using our experimental values on the two-photon absorption coefficient for Herasil glass, ${ }^{7}$ we can estimate the light intensity value entering the volume inside the sample under high voltage as $40 \mathrm{GW} / \mathrm{cm}^{2}$ (the pulse energy, entering the poling volume, is about $40 \mu \mathrm{J})$. The total irradiation fluence regarding the poling volume was about $2 \mathrm{~kJ} / \mathrm{cm}^{2}$.

A digital oscilloscope LT372 (LeCroy) was used to measure the photoelectric current induced in the sample. Immediately, at the start of the irradiation, a strong photocurrent pulse with an amplitude of up to a $20 \mathrm{~V}$ appeared, which corresponded to a conductivity value of $\sigma=2$ $\times 10^{-9} \mathrm{~cm}^{-1} \Omega^{-1}$ (resistivity value $\rho=5 \times 10^{8} \mathrm{~cm} \Omega$, which is about ten orders less than the standard reference value for room temperature $\rho=10^{18} \mathrm{~cm} \Omega^{10}$ ). The calculated initial quantum yield of charged particles between the electrodes was about $2 \times 10^{-6}$, if we presume that the time resolution of our oscilloscope was $50 \mathrm{~ns}$. During the irradiation, the pulse amplitude value degraded rather quickly (during some seconds) to a level of some volts (Fig. 2). A horizontal movement of the sample with respect to the laser beam led to a revival of the photocurrent signal. After switching off the applied voltage, the photocurrent pulse changed its polarity and continued to degrade. No current was observed in the absence of high-intensity UV light. The observed photocurrent behavior is qualitatively consistent with the one induced 


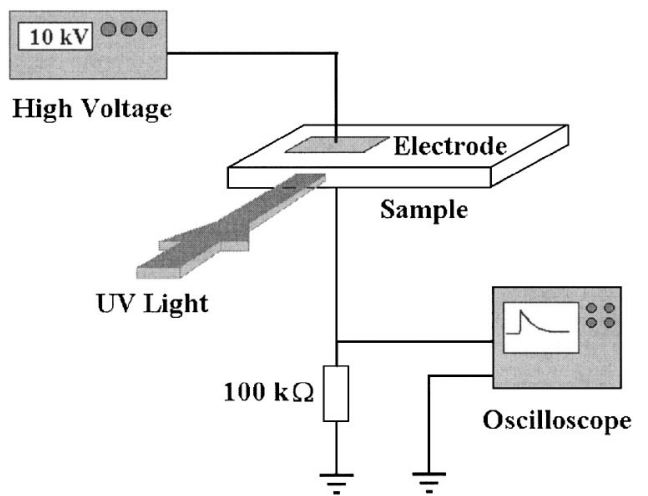

FIG. 1. Schematic of UV poling experiment with induced photocurrent measurement.

in earlier experiments on the two-step ionization $S_{0} \rightarrow S_{1}$ $\rightarrow S_{N}$ of oxygen deficient fused silica by $\mathrm{KrF}$ excimer laser radiation with $\lambda=248 \mathrm{~nm}$ and $\tau=25 \mathrm{~ns}^{11}$

After poling and removal of the electrodes (by etching), the sample was tested for evidence of second-harmonic generation. As a source of fundamental radiation, a mode-locked and Q-switched Nd:YAG laser was used $(\lambda=1064 \mathrm{~nm})$. For the Maker's fringe measurement, ${ }^{12}$ the sample was placed on a rotation stage and the laser beam was focused on the poled area, with the polarization parallel to the plane of incidence. The generated SH light was detected with a photomultplier. The measurement of the periodicity of the fringes in the Maker's fringe pattern allows us to determine the thickness of the poled region in the sample. The fit to the experimental data (Fig. 3) reveals that the region with an induced $\chi^{(2)}$ nonlinearity corresponds to the whole bulk volume between the two electrodes. The comparison of the $\mathrm{SH}$ signal value with that obtained from the reference sample (crystalline quartz) with a known $\chi^{(2)}$ value of $0.6 \mathrm{pm} / \mathrm{V},{ }^{13}$ allowed us to estimate the maximum value of the induced second-order nonlinearity as about $0.02 \mathrm{pm} / \mathrm{V}$. A rectification model $^{14}$ gives for the induced second-order nonlinearity a value of

$$
\chi^{(2)}=3 \chi^{(3)} E_{\mathrm{dc}},
$$

where $\chi^{(3)}$ is the third-order nonlinear susceptibility, equal to $4 \times 10^{-22} \mathrm{~m}^{2} \mathrm{~V}^{-2}$ for fused silica, ${ }^{15}$ and $E_{\mathrm{dc}}$ is the applied electric field. If we take $E_{\mathrm{dc}}$ equal to $200 \mathrm{kV} / \mathrm{cm}$, we will

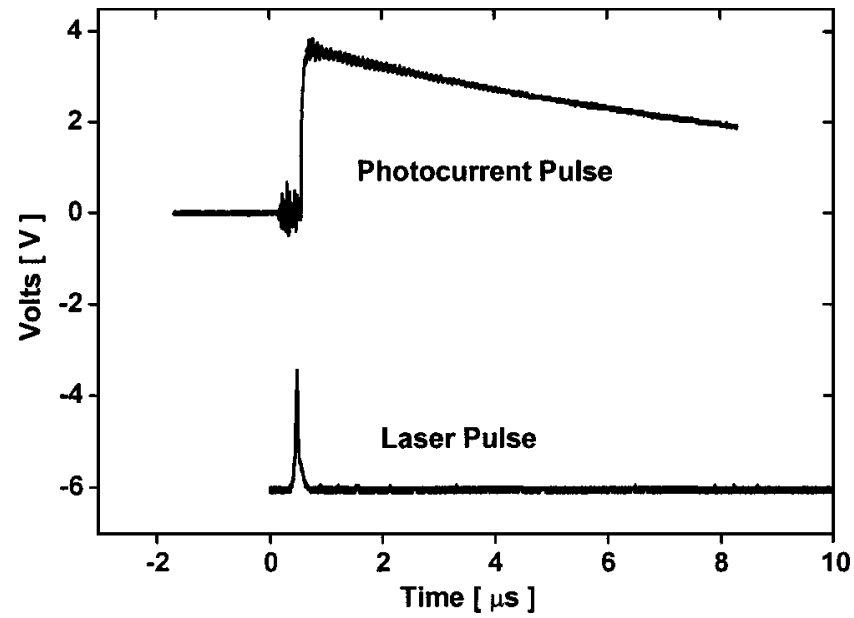

FIG. 2. Oscillogram of an induced photocurrent signal together with fem-

The UV beam entered the sample from the left.
tosecond UV pulse.
Downloaded 10 Nov 2009 to 152.78.208.72. Redistribution subject to AIP license or copyright; see http://apl.aip.o

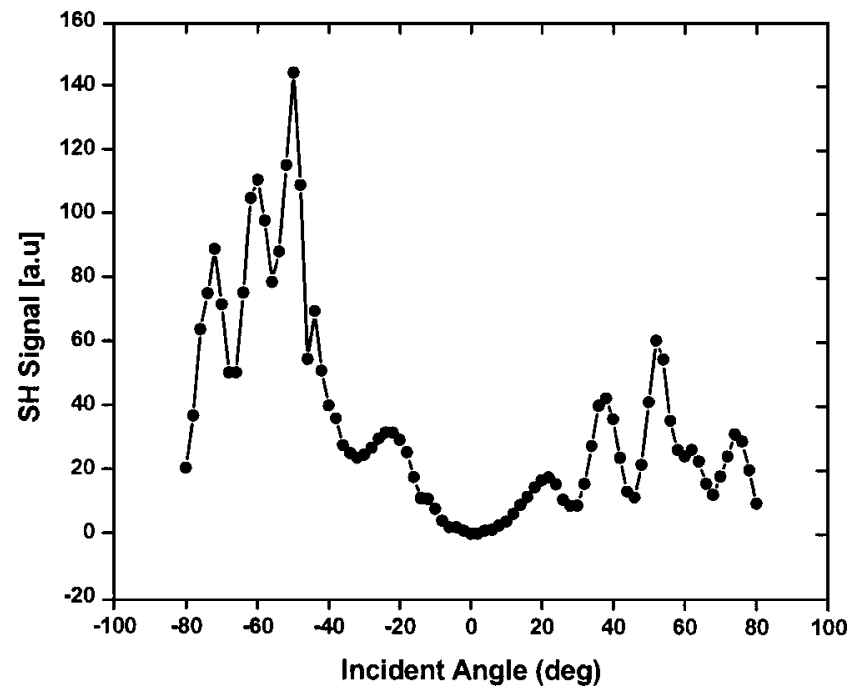

FIG. 3. Maker's fringe pattern registered in the poled sample of Herasil glass.

obtain a predicted $\chi^{(2)}$ value of $0.024 \mathrm{pm} / \mathrm{V}$, which is in remarkable agreement with the measured one. If the same experiment is performed in twin-hole fibers, ${ }^{16}$ where the maximum applied field would be only limited by the dielectric strength of silica at room temperature $\left(>3 \times 10^{8} \mathrm{~V} / \mathrm{m},{ }^{17}\right.$ $\sim 7 \times 10^{8} \mathrm{~V} / \mathrm{m},{ }^{18}>10^{9} \mathrm{~V} / \mathrm{m}^{16}$ ), we can tentatively predict a value of $\chi^{(2)}$, reaching $1 \mathrm{pm} / \mathrm{V}$.

We also scanned the pump beam across the surface of the sample to test the distribution of the second-order nonlinearity, using the setup described in our earlier work $^{19}$ (Fig. 4). The geometry was the same as for the Maker's fringe measurements described earlier. The angle of incidence was chosen to be close to normal incidence $\left(10^{\circ}\right)$ to reduce the effects of nonuniformities. A stronger SH signal is observed near the side where the laser beam entered the sample which could be explained by the decrease of UV intensity caused by two-photon absorption in the glass. During the inscription procedure, we poled several regions under the electrodes by

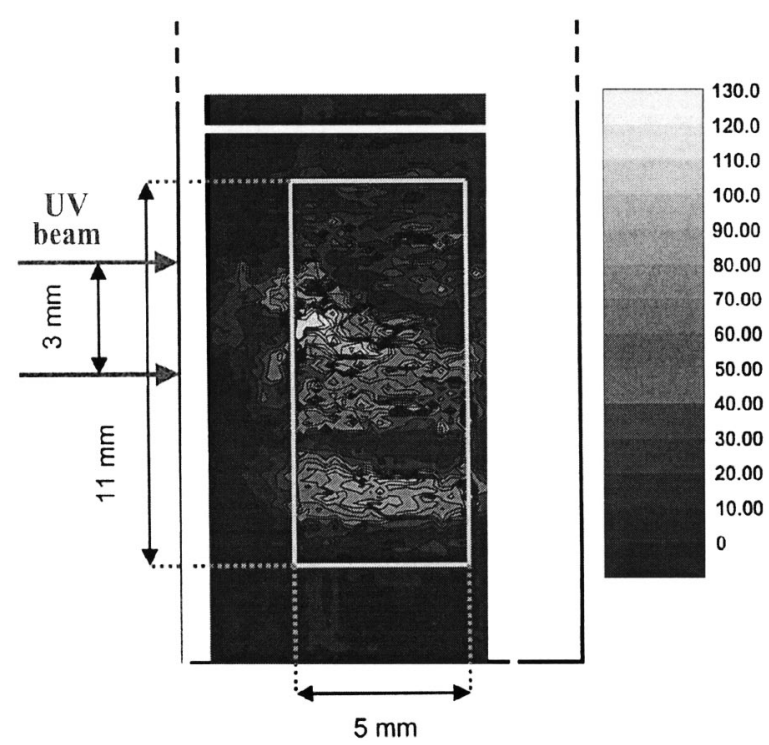

FIG. 4. The two-dimensional distribution of the second-harmonic signal in the poled fused silica sample. The sample geometry is marked by the black lines and the area covered by the electrodes is marked by the white lines. The UV beam entered the sample from the left. 
translating the sample. It is clearly seen that the secondharmonic signal is observed only in the area under the electrodes illuminated by the UV light beam, indicating that the poling takes place only under the simultaneous action of both UV light and the applied electric field.

In conclusion, using high-intensity $\left(\sim 40 \mathrm{GW} / \mathrm{cm}^{2}\right)$ femtosecond UV light combined with a high electric field (200 $\mathrm{kV} / \mathrm{cm}$ ) we were able to induce a second-order nonlinearity of $0.02 \mathrm{pm} / \mathrm{V}$ in undoped fused silica.

The authors want to thank Dr. Alastair Glass for bringing their attention to this interesting area and for useful discussions. Christie Roche and John Lucy are acknowledged for excellent technical assistance and the Science Foundation Ireland for their generous financial support (Grant No. 0.1/ IN.1/IO58).

${ }^{1}$ T. Fujiwara, M. Takahashi, and A. J. Ikushima, Appl. Phys. Lett. 71, 1032 (1997).

${ }^{2}$ T. Fujiwara, M. Takahashi, and A. J. Ikushima, Electron. Lett. 33, 980 (1997).

${ }^{3}$ M. Ohama, T. Fujiwara, and A. J. Ikushima, Jpn. J. Appl. Phys., Part 1 38, 6359 (1999).

${ }^{4}$ T. Fujiwara, S. Matsumoto, M. Ohama, and A. J. Ikushima, J. Non-Cryst.
Solids 273, 203 (2000).

${ }^{5}$ W. Margulis, F. C. Garcia, E. N. Hering, L. C. Guedes Valente, B. Lesche, F. Laurell, and I. C. S. Carvalho, MRS Bull. 23, 31 (1998).

${ }^{6}$ W. Hu, P. Blaszkiewicz, and S. Fleming, Adv. Mater. (Weinheim, Ger.) 13, 1014 (2001).

${ }^{7}$ A. Dragomir, J. G. McInerney, D. N. Nikogosyan, and P. G. Kazansky, Appl. Phys. Lett. 80, 1114 (2002).

${ }^{8}$ A. Dragomir, J. G. McInerney, and D. N. Nikogosyan, Appl. Opt. 41, 4365 (2002).

${ }^{9}$ Twinkle, highly integrated pico/femtosecond CPA Nd:glass laser system, http://www.lightcon.com/lc/scientific/laser.htm.

${ }^{10}$ Quartz Glass for Optics, Data and Properties (Heraeus Quarzglas, 1994). ${ }^{11}$ V. N. Bagratashvili, S. I. Tsypina, P. V. Chernov, A. O. Rybaltovskii, Y. S. Zavorotny, S. S. Alimpiev, Y. O. Simanovskii, L. Dong, and P. S. J. Russell, Appl. Phys. Lett. 68, 1616 (1996).

${ }^{12}$ J. Jerphagnon and S. K. Kurtz, J. Appl. Phys. 41, 1667 (1970).

${ }^{13}$ D. N. Nikogosyan, Properties of Optical and Laser-Related Materials. A Handbook (Wiley, Chichester, 1997), p. 167.

${ }^{14}$ P. G. Kazansky and P. S. J. Russell, Opt. Commun. 110, 611 (1994).

${ }^{15}$ F. Kaizar and J. Messier, Rev. Sci. Instrum. 58, 2081 (1987).

${ }^{16}$ P. G. Kazansky and V. Pruneri, Phys. Rev. Lett. 78, 2956 (1997).

${ }^{17}$ N. Myren, H. Olsson, L. Norin, N. Sjödin, P. Helander, J. Svennebrink, and W. Margulis, Opt. Express 12, 6093 (2004).

${ }^{18}$ G. W. McLellan and E. B. Strand, Glass Engineering Handbook, 3rd ed. (McGraw-Hill, New York, 1984), pp. 2-45.

${ }^{19}$ C. Corbari, J. D. Mills, O. Deparis, B. G. Klappauf, and P. G. Kazansky, Appl. Phys. Lett. 81, 1585 (2002). 